
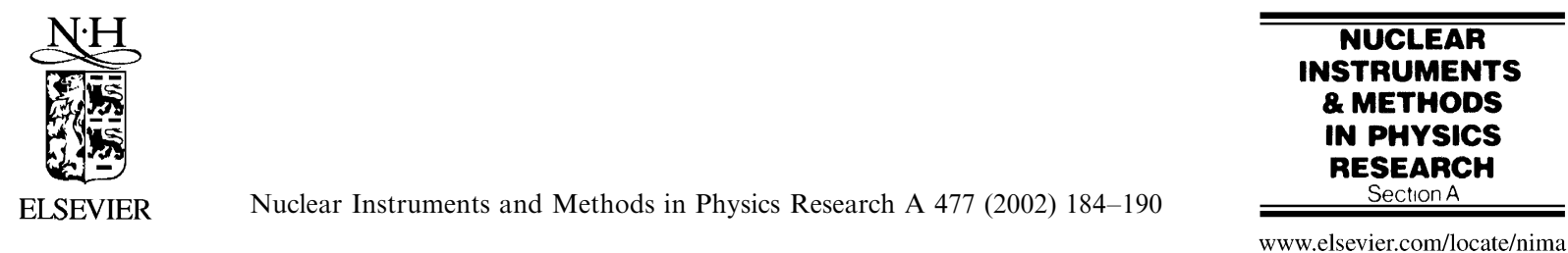

\title{
Two-dimensional readout in a liquid xenon ionisation chamber
}

\author{
V. Solovov, V. Chepel*, A. Pereira, M.I. Lopes, R. Ferreira Marques, \\ A.J.P.L. Policarpo \\ LIP-Coimbra and Departamento de Física da Universidade de Coimbra, 3000 Coimbra, Portugal
}

\begin{abstract}
A two-dimensional readout with metal strips deposited on both sides of a glass plate is investigated aiming to assess the possibility of its use in a liquid xenon ionisation chamber for positron emission tomography. Here, we present results obtained with an $\alpha$-source. It is shown that position resolution of $\leqslant 1 \mathrm{~mm}$, fwhm, can be achieved for free charge depositions equivalent to those due to $\gamma$-rays with energy from 220 down to $110 \mathrm{keV}$. (C) 2002 Elsevier Science B.V. All rights reserved.
\end{abstract}

PACS: $29.40 . \mathrm{Gx} ; 29.40 . \mathrm{Ym} ; 87.59 . \mathrm{V}$

Keywords: Liquid xenon; Ionisation chamber; Gamma-ray detection; PET

\section{Introduction}

A liquid xenon ionisation chamber triggered by xenon scintillation has been proposed as a $\gamma$-ray detector for positron emission tomography (PET) [1,2]. A prototype chamber containing about $300 \mathrm{~cm}^{3}$ of liquid has been built and studied [3-7].

The present design of the chamber is shown in Fig. 1. Very shortly, it operates as follows. The scintillation is detected by two photomultipliers. Their signals are used for coincidences and trigger the data acquisition system. The electrons released due to ionisation are collected in the central plane of wires. The collection time of the electrons is measured. It is proportional to $x$-coordinate (transaxial coordinate, speaking in terms of

*Corresponding author. Tel.: + 351-239-833465; fax + 351239-822358.

E-mail address: vitaly@lipc.fis.uc.pt (V. Chepel). tomography) that allows to measure $x$ with resolution of $0.8 \mathrm{~mm}$, fwhm.

The wires are connected in pairs and each pair is read with a charge-sensitive preamplifier. The pair of wires to which the charge is collected is identified thus determining the $z$-coordinate (depth of interaction, DOI) with precision of $5 \mathrm{~mm}$ (double wire pitch). Amplitude of the charge signal allows the deposited energy also to be measured with a resolution of $17 \%$.

The third, $y$-coordinate, can be measured by Anger method with two photomultipliers, as it was planned initially. However, the resolution provided by this method is incomparably worse $(\sim 10 \mathrm{~mm})$ than that along $x$-direction. Therefore, in the present paper we investigate an alternative method of measurement of $y$ and $z$ coordinates using ionisation signals, by replacing the plane of wires with a glass plate which has metal strips deposited on both its sides. 


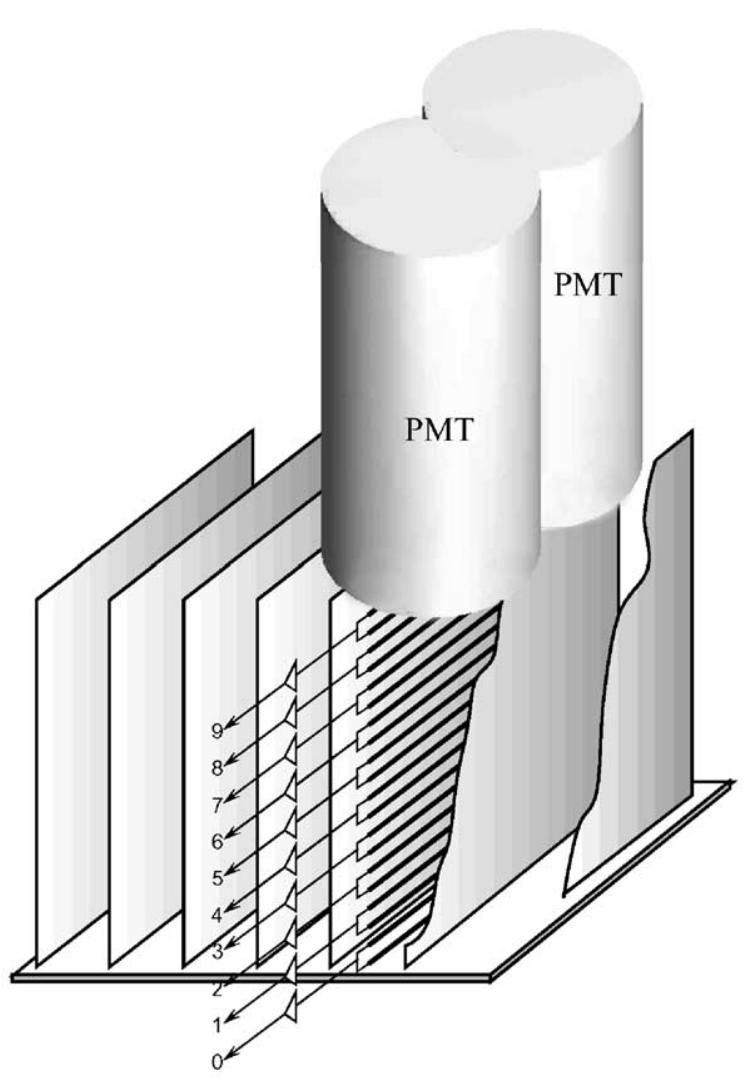

Fig. 1. Schematic drawing of the liquid xenon PET chamber.

\section{Mini-strip plate}

The original idea was developed and tested by the ICARUS group at CERN [8]. It was aimed to substitute a set of multiwire electrodes in the large liquid argon time projection chamber (TPC) by a more rigid and reliable construction. Basically, the idea was to use a printed circuit board with metal strips. Several designs were developed, some of them employing multilayer boards made from G10 with holes or grooves.

In view of our application, two important differences have to be kept in mind. Firstly, liquid xenon is known to be much more difficult to purify then argon. Impurities in very small quantities of the order of $0.1 \mathrm{ppb}$ can both reduce significantly the electron life time and result in absorption of the scintillation light. Moreover, due to smaller

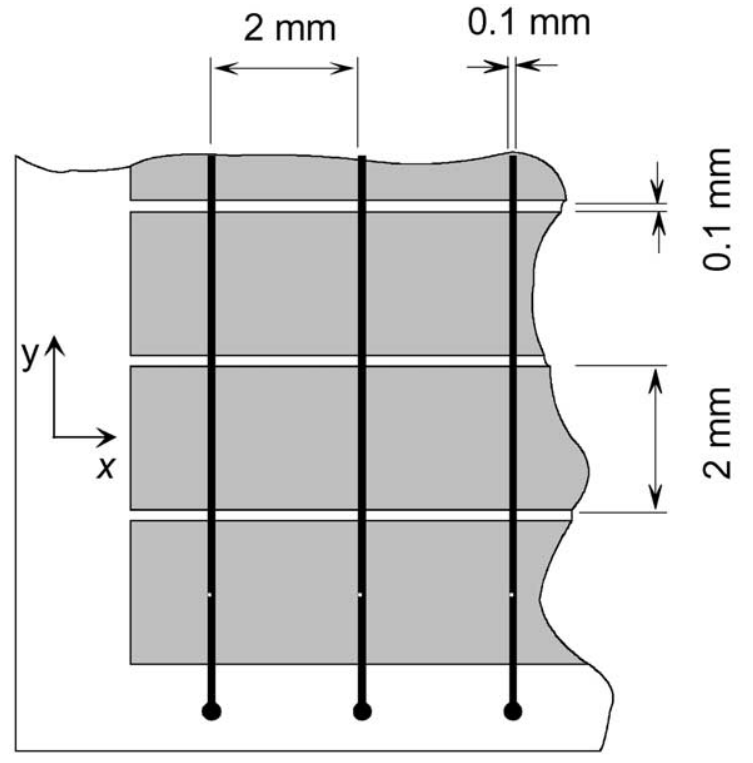

Fig. 2. Layout of the glass mini-strip plate.

volume and high segmentation of the PET detector, the ratio of the open surface of the insulator (i.e., the outgassing surface) to the volume of working material is by a factor of $\sim 100$ larger in our chamber than in the liquid argon TPC. For these reasons, we used glass for the plate substrate.

Secondly, the energy threshold accepted by the ICARUS experiment is by about an order of magnitude higher then in case of PET.

Taking into account these two aspects, it is clear that the previous experience cannot be directly applied to our conditions.

The layout of the "mini-strip" plate, as we call it for sake of briefness, is shown in Fig. 2. It is made of $50 \times 50 \mathrm{~mm}^{2}$ glass (Desag D263) $0.55 \mathrm{~mm}$ thick. The collecting strips are $0.1 \mathrm{~mm}$ wide with the pitch of $2 \mathrm{~mm}$. The induction strips (on the back side of the plate) are $1.9 \mathrm{~mm}$ wide with $0.1 \mathrm{~mm}$ space between them. They are perpendicular to the collectors. The strips were made by deposition of chromium $(\sim 0.3 \mu \mathrm{m}$ thick) on the glass substrate in vacuum. The wide strips were deposited using a mechanical mask. For the thin strips, the conventional etching technology was used. All the work has been done in our laboratory. 


\section{Method}

\subsection{Experimental}

The anode wires were replaced by the mini-strip plate in one of the cells of the chamber. Five strips from each side of the plate were used in the present measurements. Each strip was read with a TOTEM charge-sensitive preamplifier followed by a linear amplifier [9]. The signals from the cathode were also read. A thin ${ }^{241} \mathrm{Am} \alpha$-source was mounted on the cathode placed at $5 \mathrm{~mm}$ from the mini-strip plate (Fig. 3). As the active area of the source is $\varnothing 8 \mathrm{~mm}$, a mask with the hole of $\varnothing 1.5 \mathrm{~mm}$ was put on top of it. The mask was made of $0.1 \mathrm{~mm}$ thick stainless-steel foil. The resulting count rate of $\alpha$-particles was $\sim 10 \mathrm{~s}^{-1}$.

All signals were digitised by peak ADCs and the data stored in a computer. The data acquisition system was similar to that described in Ref. [5]. Flash ADCs [10] were employed in some measurements to study the signal shape. The whole system was triggered by the xenon scintillation. All charge reading channels were calibrated with respect to their charge sensitivity.

The collecting strips were kept at ground potential (including those that were not read); a negative voltage, $U_{\mathrm{c}}$, was applied to the cathode, being typically between -300 and $-2000 \mathrm{~V}$. The potential of the induction strips, $U_{\mathrm{i}}$, was varied between 0 and $-1000 \mathrm{~V}$.

An important point for our measurements is that the recombination along $\alpha$-particle tracks is very significant in liquid xenon (on the contrary to electrons and $\gamma$-rays) and strongly depends on the electric field strength. For example, the charge extracted from the track of a $5.15 \mathrm{MeV} \alpha$-particle in the field of $2000 \mathrm{~V} / \mathrm{cm}$ is only about $2.4 \mathrm{fC}$ [11], i.e. 15,000 electrons. Taking into account the $w$-value of liquid xenon, $15.6 \mathrm{eV}$ [12], the same amount of charge would be deposited by $\mathrm{a} \approx 260 \mathrm{keV}$ electron or $\gamma$-ray, $90 \%$ of which escape the recombination [13]. Therefore, the signal amplitudes that we measure with the $\alpha$-particles are similar to those we would have due to $\gamma$-rays with the energy of several hundreds $\mathrm{keV}$. Moreover, one can vary the "effective" deposited energy by changing the extracting field. In the present

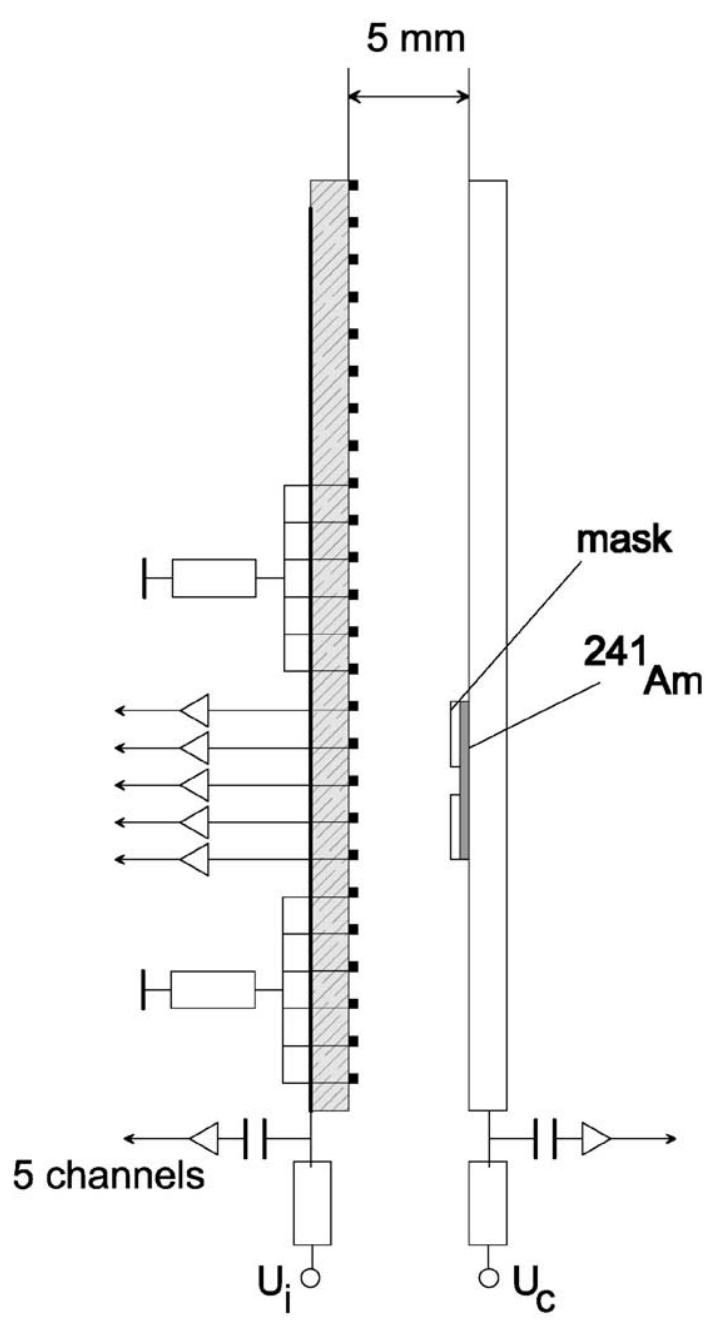

Fig. 3. A chamber cell with the mini-strip plate installed.

measurements, we could vary it between 220 and $110 \mathrm{keV}$.

\subsection{Computations}

Computations of the drift field for different voltages applied to the electrodes were carried out with a commercial 3D electrostatic solver ${ }^{1}$. The mini-strip plate configuration and the chamber

\footnotetext{
${ }^{1}$ Coulomb v4.0, Integrated Engineering Software, Inc., Canada.
} 
geometry was as described above; the dielectric permitivities of 6.4 and 1.9 were assumed for D263 glass and liquid xenon, respectively. In order to calculate the charge induced by drifting electrons to the strips, the weighting fields were computed for both collecting and induction strips, as well as the drift field in the chamber. They were used as input for a code written by us that traces an electron in liquid xenon along the field lines and computes the charge induced on the strips as a function of time [14]. The space distribution of the free electrons produced by an $\alpha$-particle was approximated by a point as its range in liquid xenon is only few tens of $\mu \mathrm{m}$. Thus, the shape of the signals from both collecting and induction strips can be computed as a function of applied voltages and position of the ionisation point due to $\alpha$-particles.

\section{Results}

To ensure the full charge collection to the collecting strips, a negative voltage has to be applied to the back strips. This is illustrated in Fig. 4, which shows the computed electron trajectories for two voltages $\left(U_{\mathrm{c}}=-1000 \mathrm{~V}\right.$ in both cases). Fig. 4 also shows the equipotential lines of the weighting field of a back strip in the plane perpendicular to the collecting strips and passing through the middle of the induction strip, which runs along $x$-direction. These lines allow the determination of the charge induced on the back strip by an electron (its charge is assumed to be equal to 1) located at different points of the plane. One can see that for $U_{\mathrm{i}}=-500 \mathrm{~V}$, for example, the induction signal with the amplitude from 0.35 up to 0.55 can be expected on the back strips.

Fig. 5 shows the computed variation of the amplitude of the charge signal induced on the back strip (centred at $y=0$ ) by an electron which starts drifting at $(0, y)$ close to the cathode.

Fig. 6 shows the experimentally obtained charge signals (due to the same $\alpha$-particle) on the collecting strip and on the induction strip that exhibits the largest signal, and compares them with the computed ones. The agreement is rather good except when the signals change too fast to be
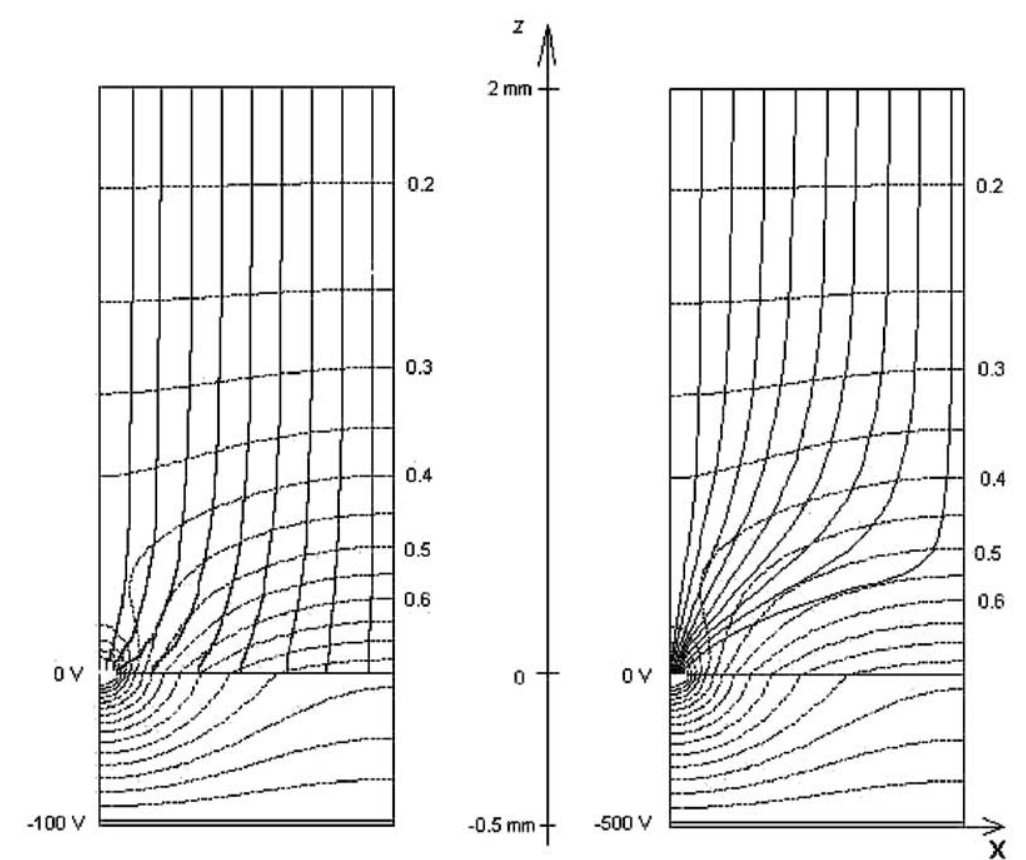

Fig. 4. Electron trajectories and equipotential lines for weighting field of an induction strip at $U_{\mathrm{c}}=-1000 \mathrm{~V}$ and $U_{\mathrm{i}}=-100 \mathrm{~V}$ (a) and $U_{\mathrm{i}}=-500 \mathrm{~V}(\mathrm{~b})$. 
followed by our slow electronics (its rise time is about $200 \mathrm{~ns}$ ).

As every drifting electron induces signals on several back strips, a centre of gravity method can be applied to determine the $y$-coordinate. The result is shown in Fig. 7 for the equivalent gammaray energies of 220 and $110 \mathrm{keV}$ and two algorithms. In one case, the signals of all induction strips were used to calculate the weighted average $y=\frac{\sum y_{\mathrm{i}} A_{\mathrm{i}}}{\sum A_{\mathrm{i}}}$

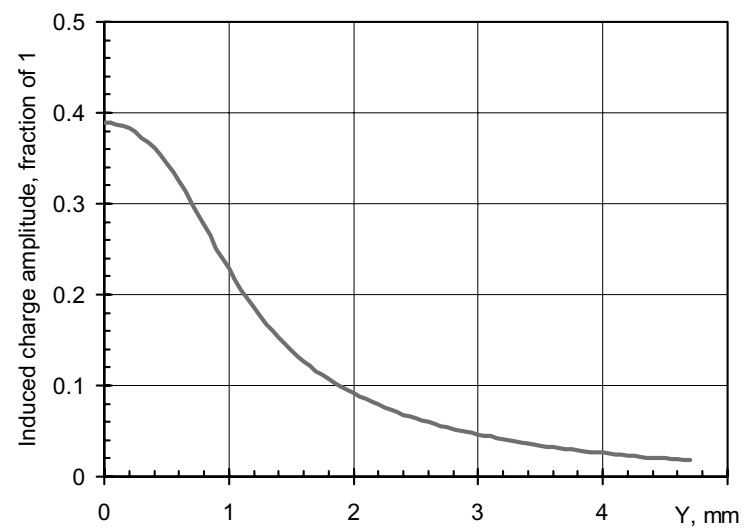

Fig. 5. Amplitude of the signal induced on the back strip centered at $y=0$ as a function of $y$, where $(x, y)$ are the coordinates of the starting point of an electron at the cathode. Computed for $x$ corresponding to the center of a collecting strip. where $A_{\mathrm{i}}$ is the amplitude of the signal which is read at the induction strip centred at $y_{\mathrm{i}}$. The second histogram was obtained with a similar method but a threshold for each signal has been introduced. The resolution as a function of "effective" deposited energy obtained with these two algorithms is shown in Fig. 8. As one should expect, the resolution is better for the algorithm with threshold and it gets worse with decreasing energy.

Concerning the position information along the $x$-direction, we can only speak of localisation precision equal to the spacing between collection strips, i.e. $2 \mathrm{~mm}$, as the signal of appreciable amplitude appears only in one channel. The signals induced on the neighbouring strips are practically undistinguishable from the noise.

\section{Conclusion}

It was shown that the mini-strip plate can be operated in liquid xenon as a 2D ionisation readout device. In the experiments carried out with an $\alpha$-source, we achieved position resolution of $\leqslant 1 \mathrm{~mm}$, fwhm, along one direction and a localisation accuracy of $2 \mathrm{~mm}$ along the other. These results were obtained with the number of free ion pairs which is equivalent to that created in liquid xenon by $\gamma$-rays with energy between

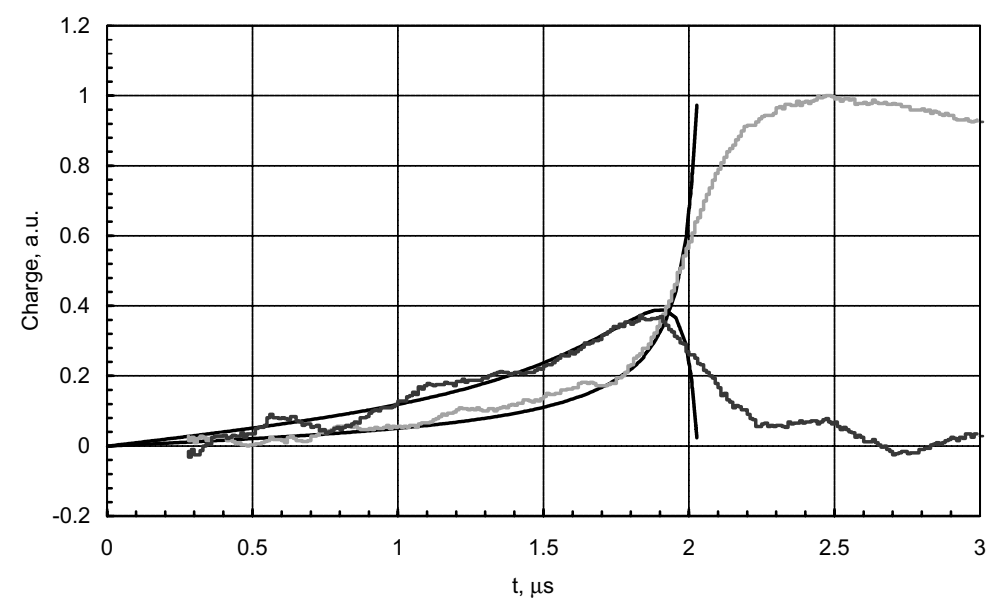

Fig. 6. Acquired signals at a collection and an induction strips, compared with the computed waveforms $\left(U_{\mathrm{c}}=-1500 \mathrm{~V}\right.$, $\left.U_{\mathrm{i}}=-500 \mathrm{~V}\right)$. 

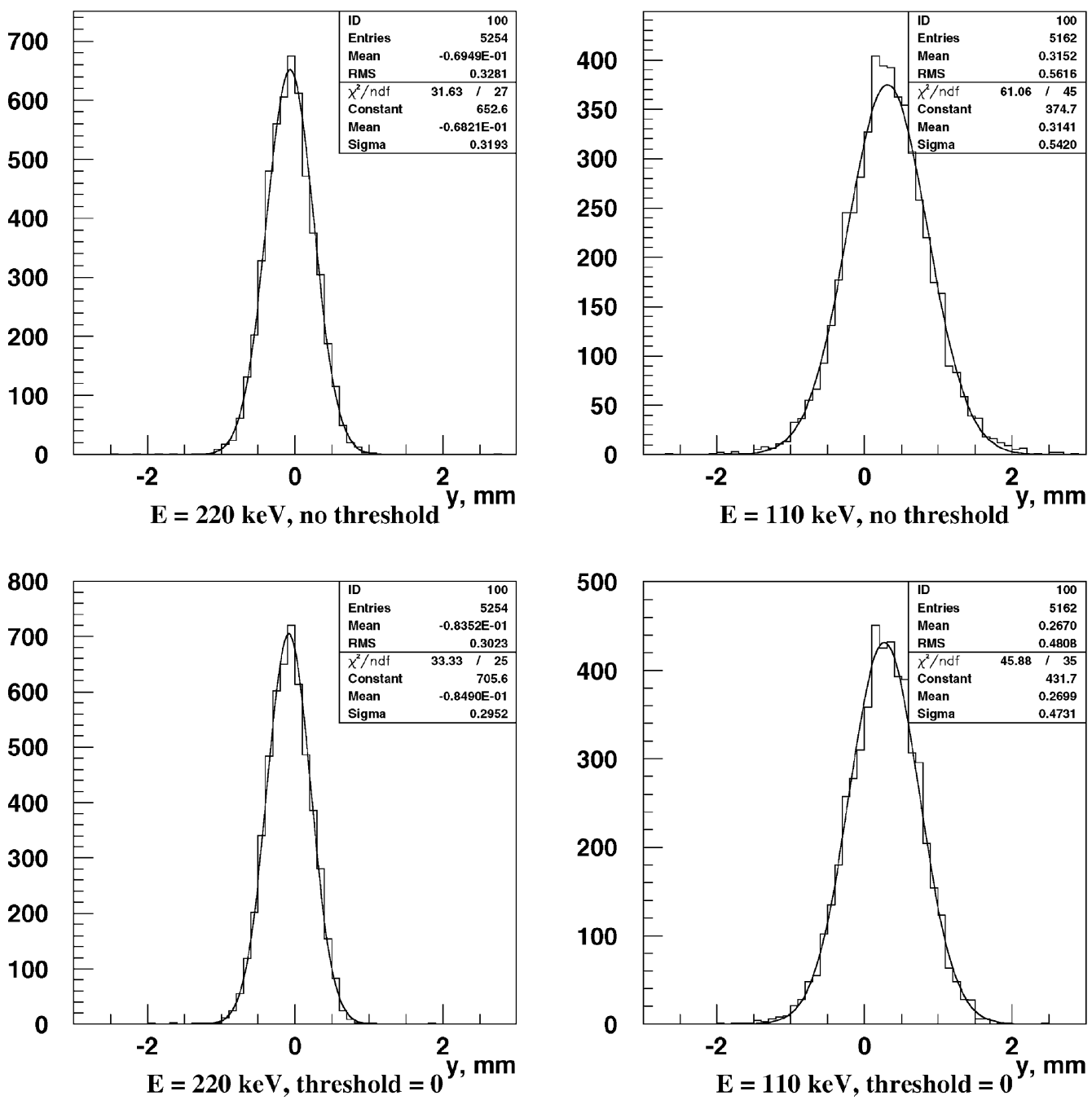

Fig. 7. Distribution of the $y$-coordinate obtained with two selection rules (see text) for equivalent gamma-ray energies of $220 \mathrm{keV}$ (left column) and $110 \mathrm{keV}$ (right column).

110 and $220 \mathrm{keV}$. As the range of photoelectrons produced by $\gamma$-rays with the energy $\leqslant 500 \mathrm{keV}$ in liquid xenon is $<0.5 \mathrm{~mm}$, practically the same precision can be expected in case of $\gamma$ photons.

The application of this readout device in the liquid xenon PET detector, which is under development, can provide excellent $(\sim 1 \mathrm{~mm})$ transaxial and axial position precision and measuring the depth of interaction (DOI) with accuracy of $\sim 2 \mathrm{~mm}$.

The fact that good position resolution was obtained with the equivalent gamma-ray energy as low as $110 \mathrm{keV}$, allows us to think about a liquid xenon 2D imaging device (gamma-camera) with purely ionisation readout. To achieve similarly good resolution in both directions, the influence of the plate parameters on the system performance 


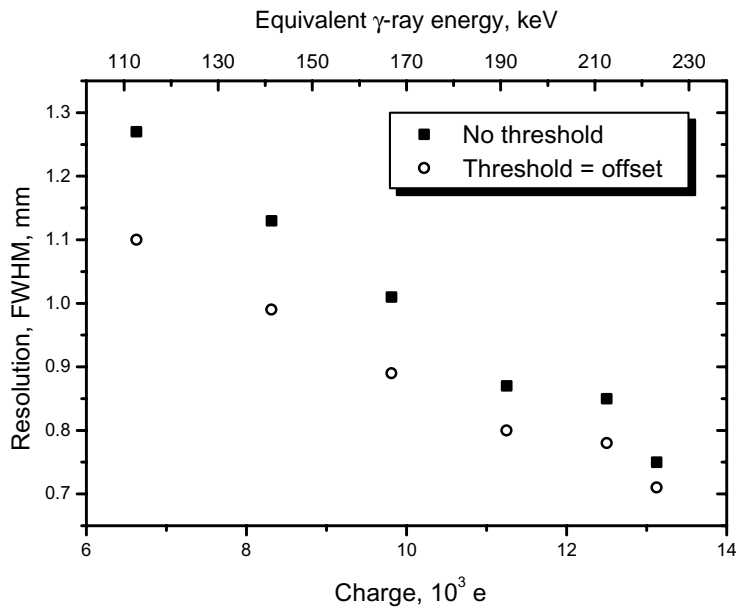

Fig. 8. Position resolution along $y$-direction as a function of collected charge (equivalent gamma-ray energy).

has to be better studied and different plates should be tested.

\section{Acknowledgements}

The authors wish to thank P. Picchi, F. Pietropaolo (ICARUS group at CERN) and P. Cennini (CERN) for useful discussions and suggestions, as well as L. Margato from University of Minho for his help with spattering.
The work was financed in parts by the projects PRAXIS XXI /2./2.1/SAU/1342/95 and PBIC/C/ FIS/2214/95 from the Fundação para Ciência e Tecnologia, Portugal. One of the authors was supported by a fellowship PRAXIS XXI /BD/ $3892 / 96$ from the same organisation.

\section{References}

[1] V.Yu. Chepel, Nucl. Tracks Radiat. Meas. 21 (1993) 47.

[2] M.I. Lopes, et al., IEEE Trans. Nucl. Sci. NS-42 (1995) 2298.

[3] V.Yu. Chepel, et al., Nucl. Instr. and Meth. A 367 (1995) 58.

[4] V.Yu. Chepel, et al., Nucl. Instr. and Meth. A 397 (1997) 427.

[5] P. Crespo, et al., IEEE Trans. Nucl. Sci. NS-47 (2000) 2119.

[6] V. Chepel, et al., IEEE Trans. Nucl. Sci. 46 (1999) 1038.

[7] M.I. Lopes, et al., Proceedings of the 13th International Conference on Dielectric Liquids, Nara, Japan, IEEE Cat. No 99CH36213, July 1999, p. 307.

[8] P. Cennini, et al., Nucl. Instr. and Meth. A 346 (1994) 550.

[9] P. Benetti, et al., Nucl. Instr. and Meth. A 332 (1993) 395.

[10] V. Commichau, FADC V02C, III. Physikalisches Institute, RWTH Aachen, 1984 (Internal Report).

[11] E. Aprile, et al., Nucl. Instr. and Meth. A 307 (1991) 119.

[12] T. Takahashi, et al., Phys. Rev. A 12 (1975) 1771.

[13] E. Aprile, et al., Nucl. Instr. and Meth. A 302 (1991) 177.

[14] S. Ramo, Proc. IRE 27 (1939) 584. 